\title{
SOLVING CONTINUOUS-DOMAIN PROBLEMS EXACTLY WITH MULTIRESOLUTION B-SPLINES
}

\author{
Thomas Debarre, Julien Fageot, Harshit Gupta, and Michael Unser*
}

Biomedical Imaging Group, École polytechnique fédérale de Lausanne, Switzerland

\begin{abstract}
We propose a discretization method for continuous-domain linear inverse problems with multiple-order total-variation (TV) regularization. It is based on a recent result that proves that such inverse problems have sparse polynomial-spline solutions. Our method consists in restricting the search space to splines with knots on a uniform grid, which results in a standard convex finite-dimensional problem. As basis functions for this search space, we use the B-splines matched to the regularization order, which are optimally localized. This leads to a well-conditioned, computationally feasible optimization task. Our proposed iterative multiresolution algorithm then refines the grid size until a desired level of accuracy is met and converges to sparse solutions of our inverse problem. Finally, we present experimental results that validate our approach.
\end{abstract}

Index Terms - B-splines, inverse problems, total variation, sparsity, compressed sensing.

\section{INTRODUCTION}

In recent years, sparsity has become an increasingly important paradigm in the field of signal processing. It is at the core of compressed sensing (CS), which has flourished in the past decade [1-3]. The objective in discrete CS-type problems is to recover a signal $c \in \mathbb{R}^{N}$ given some linear measurements $\boldsymbol{y} \in \mathbb{R}^{M}$, where $M \ll N$. To overcome the ill-posedness of such an inverse problem, the signal is assumed to be sparse in a certain basis. This assumption is typically enforced with sparsity-promoting $\ell_{1}$ regularization.

For obvious considerations of computational feasibility, CS mostly deals with discrete signals; however, most models of real-world signals are continuously defined. This discrepancy can lead to discretization errors

- in the forward model (e.g., when taking the discrete Fourier transform instead of samples of the continuousdomain Fourier transform in MRI reconstructions);

- in the regularization (e.g., when using finite differences to approximate the derivative for TV regularization).

*This work was funded by an ERC grant (ERC-692726-GlobalBioIm).
To address these limitations, several works attempt to adapt the theory of discrete CS to the continuous domain [4-7]. Another prominent example is the study of inverse problems in spaces of measures [8-11]. In [12], Unser et al. introduce a framework that includes 1D continuous-domain inverse problems with $N_{0}$ th-order TV regularization. Their main result states that such inverse problems have sparse polynomial spline solutions (i.e., piecewise polynomials of degree $\left.\left(N_{0}-1\right)\right)$ with few knots.

Two main discretization strategies have been proposed to solve this type of continuous-domain problems numerically

- grid-free algorithms that solve non-convex problems [8, 13-15];

- grid-based methods which lead to convex optimization problems [16].

In this paper, we opt for a grid-based approach, as it leads to simple and effective convex-optimization algorithms. The search space of the optimization problem becomes the space of polynomial splines of order $N_{0}$ on a uniform grid. They can be represented using the Green's function $\rho_{N_{0}}$ of the $N_{0}$ th-order derivative $\mathrm{D}^{N_{0}}$ as basis function as done in [16]. Here, we improve this approach by using the B-spline $\beta_{N_{0}}$ matched to $\mathrm{D}^{N_{0}}$. Contrarily to $\rho_{N_{0}}, \beta_{N_{0}}$ has finite support, which leads to much better conditioned problems and, thus, computationally more feasible algorithms.

We first present some background information on polynomial splines and the continuous-domain framework of [12]. We then show how we discretize and solve continuousdomain problems by recasting them as standard CS-type finite-dimensional convex-optimization problem. Next, we present our multiresolution algorithm that refines the grid until a desired level of accuracy is met. Finally, we validate our framework with numerical experiments.

\section{POLYNOMIAL SPLINES}

In this work, we focus on inverse problems with $N_{0}$ th-order TV regularization. As will be clarified in Section 3, such problems are intimately related to polynomial splines.

A nonuniform polynomial spline of order $N_{0}$ is defined as a function $s$ that satisfies $\mathrm{D}^{N_{0}}\{s\}(x)=\sum_{k} a_{k} \delta\left(x-x_{k}\right)$, 
where $a_{k}, x_{k} \in \mathbb{R}$. It can be expressed as

$$
s(x)=p(x)+\frac{1}{\left(N_{0}-1\right) !} \sum_{k} a_{k}\left(x-x_{k}\right)_{+}^{N_{0}-1},
$$

where $\mathrm{D}^{N_{0}}\{p\}=0$ and $x_{+}:=\max (0, x)$. Indeed, $\rho_{N_{0}}(x)=$ $\frac{x_{+}^{N_{0}-1}}{\left(N_{0}-1\right) !}$ is the causal Green's function of $\mathrm{D}^{N_{0}}$, with $\mathrm{D}^{N_{0}}\left\{\rho_{N_{0}}\right\}=$ $\delta$. In other words, $s$ is a piecewise polynomial of degree $\left(N_{0}-1\right)$, of differentiability class $\mathcal{C}^{N_{0}-2}$, and with knots at locations $x_{k}$.

Finally, we notate the B-spline $[17,18]$ matched to $\mathrm{D}^{N_{0}}$ as

$$
\beta_{N_{0}}(x)=\frac{\Delta_{+}^{N_{0}}\left\{x_{+}^{N_{0}-1}\right\}}{\left(N_{0}-1\right) !}
$$

where $\Delta_{+}\{f\}(x):=(f(x)-f(x-1))$ is a finite-difference operator. By construction, the B-spline (2) is a cardinal polynomial spline (i.e., it has integer knots $x_{k}$ ). It has finite support in $\left[0, N_{0}\right]$. As will be elaborated in Section 4.1 , it is also a generator of the space of cardinal polynomial splines of or$\operatorname{der} N_{0}$. For a grid size $h>0$, the scaled B-spline matched to $\mathrm{D}^{N_{0}}$ is then given by $\beta_{N_{0}, h}(x)=\beta_{N_{0}}(x / h)$.

\section{CONTINUOUS-DOMAIN INVERSE PROBLEMS}

We now introduce the framework and notations of [12], which is the basis of our work. We focus on continuous-domain inverse problems, where the goal is to recover a signal $s: \mathbb{R} \rightarrow$ $\mathbb{R}$ given a finite number of measurements $\boldsymbol{y} \in \mathbb{R}^{M}$. They are acquired via a measurement operator $\nu: s \rightarrow \boldsymbol{\nu}(s) \in \mathbb{R}^{M}$. This forward model models the physics of the acquisition system (e.g., Fourier samples for MRI imaging). We thus have that $\boldsymbol{y}=\boldsymbol{\nu}(s)+\boldsymbol{n}$, where the additive noise $\boldsymbol{n} \in \mathbb{R}^{M}$ models the measurement errors.

Next, let $\mathcal{M}(\mathbb{R})$ be the space of finite Radon measures endowed with the norm $\|\cdot\|_{\mathcal{M}}$, which is the continuous counterpart of the $\ell_{1}$ norm. For the regularization, we use the $N_{0}$ thorder TV semi-norm $\|\cdot\|_{\mathrm{TV}^{\left(N_{0}\right)}}$ defined as

$$
\|f\|_{\mathrm{TV}^{\left(N_{0}\right)}}:=\left\|\mathrm{D}^{N_{0}}\{f\}\right\|_{\mathcal{M}}=\sup _{\substack{\varphi \in \mathcal{S}(\mathbb{R}) \\\|\varphi\|_{\infty}=1}}\left\langle f, \mathrm{D}^{N_{0}}\{\varphi\}\right\rangle,
$$

where $\mathcal{S}(\mathbb{R})$ is Schwartz' space of smooth and rapidly decaying functions on $\mathbb{R}$. The native space of our optimization problem is then given by $\mathcal{M}_{N_{0}}(\mathbb{R})=\left\{f \in \mathcal{S}^{\prime}(\mathbb{R})\right.$ : $\left.\|f\|_{\mathrm{TV}^{\left(N_{0}\right)}}<+\infty\right\}$, where $\mathcal{S}^{\prime}(\mathbb{R})$ is the space of tempered distributions. We now state the main result of [12] (to which we refer for the most general case), expressed in an equivalent form formulated in [16].

Theorem 1. Let $N_{0}>0$ and let $\nu: \mathcal{M}_{N_{0}}(\mathbb{R}) \rightarrow \mathbb{R}^{M}$ be a weak ${ }^{*}$-continuous ${ }^{1}$ linear measurement operator. Assume

\footnotetext{
${ }^{1}$ This is a mild albeit technical assumption. We refer to [12] for the definition, and to section 6 for some examples.
}

that $\boldsymbol{\nu}(p) \neq 0$ for all polynomials $p$ of degree less than $N_{0}$. Then, the linear inverse problem

$$
\mathcal{S}=\underset{f \in \mathcal{M}_{N_{0}}(\mathbb{R})}{\arg \min }\left\{\|\boldsymbol{\nu}(f)-\boldsymbol{y}\|_{2}^{2}+\lambda\|f\|_{\mathrm{TV}^{\left(N_{0}\right)}}\right\}
$$

has a nonempty weak ${ }^{*}$-compact convex solution set $\mathcal{S}$ whose extreme points are nonuniform polynomial splines of the form

$$
s(x)=\frac{1}{\left(N_{0}-1\right) !} \sum_{k=1}^{K} a_{k}\left(x-x_{k}\right)_{+}^{N_{0}-1}+\sum_{n=0}^{N_{0}-1} b_{n} x^{n}
$$

where the sparsity index $K$ verifies $K \leq\left(M-N_{0}\right)$.

\section{EXACT DISCRETIZATION}

\subsection{Search Space}

We now seek to discretize Problem (4) in the space of uniform polynomial splines with grid size $h$ (i.e., with knots $x_{k} \in h \mathbb{Z}$ ). This choice is guided by the form (5) of the solutions, since our search space contains functions that are arbitrarily close to the latter when $h$ is sufficiently small. Another key justification is that our search space leads to a convex optimization problem, which is much easier to solve than the original problem (4).

We introduce the search space

$$
\begin{aligned}
\mathcal{M}_{N_{0}, h}(\mathbb{R})= & \left\{\frac{1}{\left(N_{0}-1\right) !} \sum_{k \in \mathbb{Z}} a[k](\cdot-k)_{+}^{N_{0}-1}+\sum_{n=0}^{N_{0}-1} b_{n}(\cdot)^{n}\right. \\
& \left.: a \in \ell_{1}(\mathbb{Z}), b_{n} \in \mathbb{R}\right\} \\
= & \left\{\sum_{k \in \mathbb{Z}} c[k] \beta_{N_{0}, h}(\cdot-h k):\left(d_{N_{0}} * c\right) \in \ell_{1}(\mathbb{Z})\right\},
\end{aligned}
$$

where the equality is justified by the fact that uniform splines can be represented using $\rho_{N_{0}}$ or $\beta_{N_{0}, h}$ as basis functions. The space (6) satisfies $\mathcal{M}_{N_{0}, h}(\mathbb{R}) \subset \mathcal{M}_{N_{0}}(\mathbb{R})$. The sequence $d_{N_{0}}$ is characterized by its $z$ transform $D_{N_{0}}(z)=\left(1-z^{-1}\right)^{N_{0}}$. The first representation in (6) is known as the Green's function basis and is used in [16]. However, $\rho_{N_{0}}$ has an infinite support, which leads to severely ill-conditioned problems in practice. We therefore prefer the second formulation in (6) (the B-spline basis), since $\beta_{N_{0}, h}$ has finite support. It forms a Riesz basis [17], which leads to good conditioning and, thus, efficient implementations.

\subsection{Discrete Formulation}

Now that the search space (6) has been specified, solving Problem (4) in $\mathcal{M}_{N_{0}, h}(\mathbb{R})$ amounts to an infinite-dimensional discrete optimization problem over the B-spline coefficients $c[k], k \in \mathbb{Z}$. In practice, however, most real-world signals 
have the finite support $I_{T}=[0, T]$ where $T>0$. Let $I=$ $\left\{k_{\min }, \ldots, k_{\max }\right\}$ (with $N=\# I \approx T / h$ ) be the set of indices such that the support of $\beta_{N_{0}, h}(\cdot-h k)$ intersects with $I_{T}$ if and only if $k \in I$. Since there is no need to optimize over coefficients $c[k]$ such that $k \notin I$, Problem (4) restricted to the search space $\mathcal{M}_{N_{0}, h}(\mathbb{R})$ is exactly equivalent ${ }^{2}$ to the discrete problem

$$
\mathcal{S}_{h}=\underset{\boldsymbol{c} \in \mathbb{R}^{N}}{\arg \min }\left\{\|\mathbf{H} \boldsymbol{c}-\boldsymbol{y}\|_{2}^{2}+\lambda\|\mathbf{L} \boldsymbol{c}\|_{1}\right\}
$$

where the system matrix $\mathbf{H} \in \mathbb{R}^{M \times N}$ is given by

$$
\mathbf{H}=\left[\boldsymbol{h}_{k_{\min }} \cdots \boldsymbol{h}_{k_{\max }}\right]: \quad \boldsymbol{h}_{k}=\boldsymbol{\nu}\left(\beta_{N_{0}, h}(\cdot-h k)\right)
$$

and the regularization matrix $\mathbf{L} \in \mathbb{R}^{\left(N-N_{0}\right) \times N}$ by

$$
\mathbf{L}=\frac{1}{h^{N_{0}-1}}\left(\begin{array}{cccccc}
d_{N_{0}}\left[N_{0}\right] & \cdots & d_{N_{0}}[0] & 0 & \cdots & 0 \\
0 & \ddots & & \ddots & \ddots & \vdots \\
\vdots & \ddots & \ddots & & \ddots & 0 \\
0 & \cdots & 0 & d_{N_{0}}\left[N_{0}\right] & \cdots & d_{N_{0}}[0]
\end{array}\right)
$$

Note that the so-called penalized basis pursuit (PBP) problem (7) has the same form as typical discrete CS-type problem. However, there are subtle but important differences due to the fact that contrary to purely discrete frameworks, our method uses optimal basis functions that are matched to the regularization.

\subsection{Reaching a Sparse Solution}

In practice, Problem (7) can be solved using a standard convex optimization algorithm such as ADMM [19]. However, the solution set $\mathcal{S}_{h}$ is non-unique in general, whereas we only seek sparse solutions of the form (5).

To reach an extreme point of $\mathcal{S}_{h}$, we adopt a pipeline adapted from [16]:

- reach a solution $c \in \mathcal{S}_{h}$ using ADMM and compute $\boldsymbol{y}_{\lambda}=\mathbf{H} \boldsymbol{c}$

- recast Problem (7) as a linear program (for which $\boldsymbol{y}_{\lambda}$ is needed) and reach an extreme point $c^{*}$ of $\mathcal{S}_{h}$ using the simplex algorithm [20].

\section{MULTIRESOLUTION STRATEGY}

Section 4 detailed how to reach a sparse solution of Problem (4) for a fixed grid size $h$. However, the determination of an appropriate grid size highly depends on the type of problem. We address this issue by adopting now a multiresolution strategy based on grid refinement. More precisely, we take grid sizes $\left(h_{n}=T / 2^{n}\right)_{n \geq n_{0}}$, where $h_{n_{0}}$ is the starting grid size.

\footnotetext{
${ }^{2}$ under a mild condition over the measurement operator $\boldsymbol{\nu}$
}

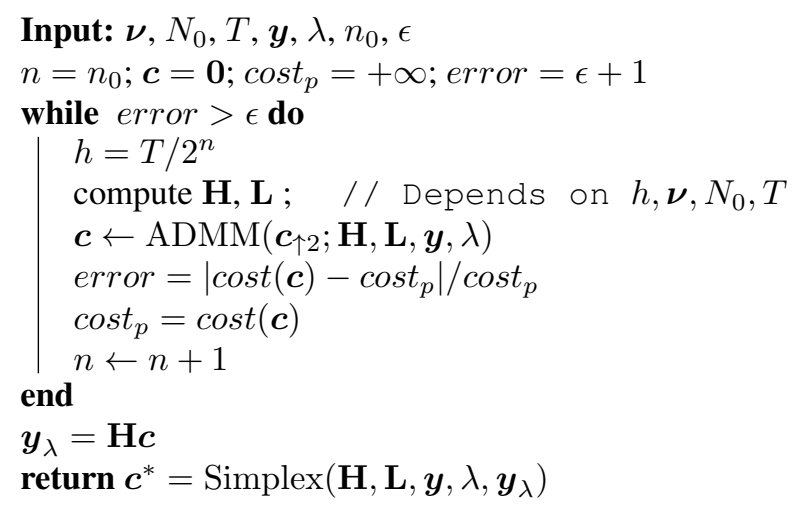

Algorithm 1: Pseudocode of our algorithm

Clearly, a spline with knots in $h_{n} \mathbb{Z}$ also has knots in $h_{n+1} \mathbb{Z}$ since $h_{n} \mathbb{Z} \subset h_{n+1} \mathbb{Z}$. This implies that the search spaces are embedded like

$$
\mathcal{M}_{N_{0}, h_{n}}(\mathbb{R}) \subset \mathcal{M}_{N_{0}, h_{n+1}}(\mathbb{R}) .
$$

This allows us to use a solution $c \in \mathcal{S}_{h_{n}}$ as a warm start for the next finer grid size, by converting it to a vector $\boldsymbol{c}_{\uparrow 2}$ that represents the same continuous-domain signal. The use of a warm start leads to a substantial acceleration of the algorithm. Finally, the embedding (10) guarantees that the cost will decrease at every grid size, so that $\mathcal{J}_{h_{n}}^{0} \geq \mathcal{J}_{h_{n+1}}^{0}$ where $\mathcal{J}_{h}^{0}$ is the optimal cost of Problem (7). As termination criterion, we stop the refinement as soon as the sequence $\left(\mathcal{J}_{h_{n}}^{0}\right)_{n \geq n_{0}}$ ceases to decrease within a certain user-defined tolerance $\epsilon>0$. Formally, this translates to $\left(\mathcal{J}_{h_{n-1}}^{0}-\mathcal{J}_{h_{n}}^{0}\right) / \mathcal{J}_{h_{n-1}}^{0}<\epsilon$.

The pseudocode of our multiresolution algorithm is given in Algorithm 1. Note that the simplex step is only required at the final grid size, since any solution (not necessarily a sparse one) can be used to compute the optimal cost $\mathcal{J}_{h_{n}}^{0}$.

\section{EXPERIMENTS}

We now show numerical applications of Algorithm 1. Our code was implemented in Matlab using GlobalBioIm [21], an inverse-problem library developed in our group, and the Gurobi optimizer $^{3}$ for the simplex step. In all our experiments, we use a tolerance $\epsilon=10^{-3}$ for the grid refinement.

In our first experiment (Figure 1), we set $N_{0}=1$ (TV regularization) with $M=10$ measurements of the form

$$
\nu_{m}(f)=f\left(x_{m}\right): \quad x_{m} \in[0, T] .
$$

This amounts to an interpolation problem using a piecewiseconstant signal. The ground-truth signal is not shown in Figure 1, only its measurements. The sparsity of the reconstructed signal is consistent with Theorem 1 (its sparsity index $K=9$ satisfies $K \leq\left(M-N_{0}\right)$ ). Observe that the coarse grid $h=1 / 2^{5}$ is sufficient to get an adequate reconstruction.

\footnotetext{
${ }^{3}$ LLC Gurobi Optimization, Gurobi optimizer reference, 2018.
} 


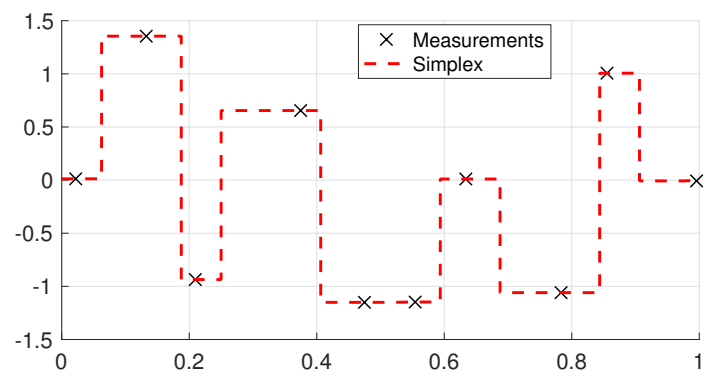

Fig. 1: Reconstructed signal for an interpolation problem with TV regularization $\left(N_{0}=1\right)$ for $M=10$ and $h=1 / 2^{5}$.

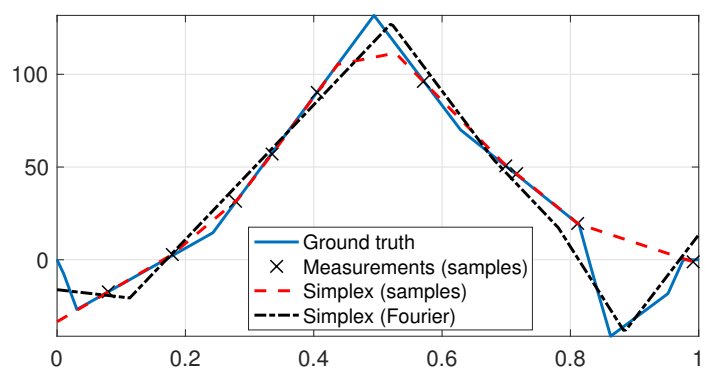

Fig. 2: Reconstructions using ideal samples $(\mathrm{SNR}=12.03$ $\mathrm{dB})$ and Fourier-domain measurements $(\mathrm{SNR}=18.07 \mathrm{~dB})$ for $N_{0}=2, M=10$ and $h=1 / 2^{8}$.

For our following experiments, we use a sparse nonuniform polynomial spline of order $N_{0}$ of the form (1) as our ground-truth signal for the $N_{0}$ th-order TV regularization to be an adequate prior. The given signal-to-noise (SNR) values quantify how faithful our reconstructed signal is to this ground-truth signal. To verify the robustness of our algorithm, we add Gaussian white noise to the measurements.

In the experiment shown in Figure 2, we set $N_{0}=2$. We use two different measurement operators: ideal samples as in (11); and Fourier-domain samples of the form

$$
\nu_{m}(f)=\int_{0}^{T} f(x) \cos \left(\omega_{m} x+\theta_{m}\right) \mathrm{d} x,
$$

where $m \in\{1, \ldots, M\}, \omega_{m} \geq 0$, and $\theta_{m} \in[0, \pi)$. We then compare the reconstructions results for both measurement operators on the same ground-truth signal with the same number $M=10$ of measurements. As predicted by the theory of CS (i.e., Fourier matrices are known to have good recovery properties [22]), the reconstruction using Fourier-domain samples is very accurate despite the small number of measurements and the presence of noise. Conversely, our reconstruction using ideal simples is less faithful since we have no information on the behavior of the signal between sampling points. This disparity is underlined by the difference in SNR values (12.03 $\mathrm{dB}$ versus $18.07 \mathrm{~dB}$ ).

Finally, in the experiment shown in Figure 3, we set $N_{0}=$ 4, with $M=10$ Fourier-domain samples of the form (12) as

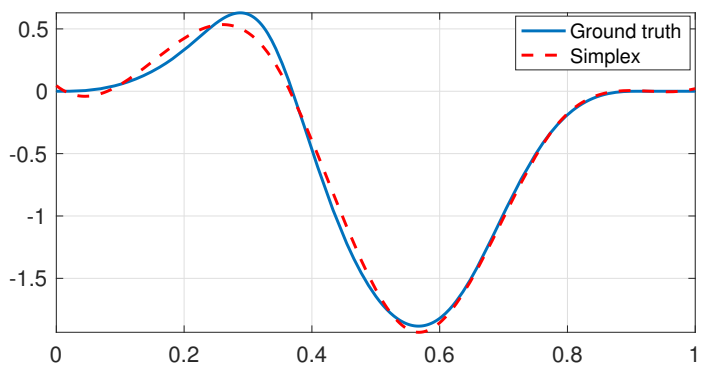

(a) Reconstructed signal for $h=1 / 2^{9}(\mathrm{SNR}=22.71 \mathrm{~dB})$.

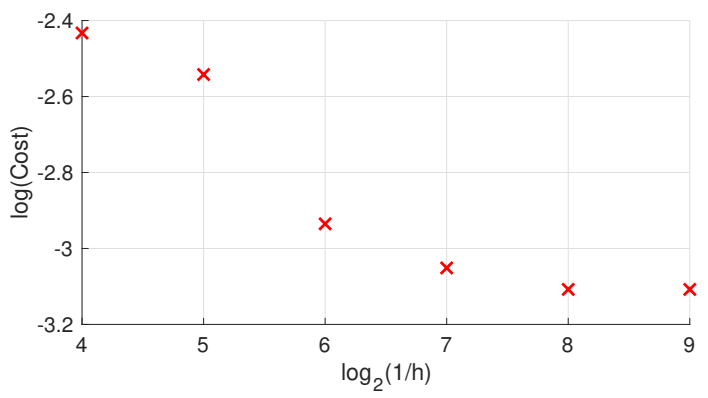

(b) Evolution of $\mathcal{J}_{h}^{0}$ with respect to $h$.

Fig. 3: Example run for $N_{0}=4$ and $M=10$ (Fourierdomain measurements).

measurements. Reconstruction results using Algorithm 1 are shown in Figure 3a. Once again, despite the small number of measurements, the reconstruction is quite accurate. The evolution of the optimal cost $\mathcal{J}_{h}^{0}$ is shown in Figure 3b. We notice that it initially decreases at every step of the grid refinement, but ceases to decrease between $h=1 / 2^{8}$ and $h=1 / 2^{9}$. This indicates that it has reached its limit value, and that there is no further need to refine the grid. Note that, due to the finite support of B-splines, Algorithm 1 is extremely efficient and typically converges in a few seconds.

\section{CONCLUSION}

We have presented an exact B-spline-based discretization method for solving continuous-domain inverse problems with gTV regularization. Our proposed multiresolution algorithm automatically selects an appropriate grid by exploiting the advantageous properties of splines. We have then validated our algorithm numerically with several examples that highlight its efficiency in terms of computation time. 


\section{References}

[1] D.L. Donoho, "Compressed sensing," IEEE Transactions on Information Theory, vol. 52, no. 4, pp. 12891306, 2006.

[2] Y.C. Eldar and G. Kutyniok, Compressed Sensing: Theory and Applications, Cambridge University Press, 2012.

[3] S. Foucart and H. Rauhut, A Mathematical Introduction to Compressive Sensing, vol. 1, Birkhäuser Basel, 2013.

[4] Y.C. Eldar, "Compressed sensing of analog signals in shift-invariant spaces," IEEE Transactions on Signal Processing, vol. 57, no. 8, pp. 2986-2997, 2009.

[5] M. Mishali, Y.C. Eldar, and A.J. Elron, "Xampling: Signal acquisition and processing in union of subspaces," IEEE Transactions on Signal Processing, vol. 59, no. 10, pp. 4719-4734, 2011.

[6] B. Adcock and A.C. Hansen, "Generalized sampling and infinite-dimensional compressed sensing," Foundations of Computational Mathematics, pp. 1-61, 2015.

[7] B. Adcock, A.C. Hansen, C. Poon, and B. Roman, "Breaking the coherence barrier: A new theory for compressed sensing," in Forum of Mathematics, Sigma. Cambridge University Press, 2017, vol. 5.

[8] K. Bredies and H.K. Pikkarainen, "Inverse problems in spaces of measures," ESAIM: Control, Optimisation and Calculus of Variations, vol. 19, no. 01, pp. 190-218, 2013.

[9] E.J. Candès and C. Fernandez-Granda, "Towards a mathematical theory of super-resolution," Communications on Pure and Applied Mathematics, vol. 67, no. 6, pp. 906-956, 2014.

[10] V. Duval and G. Peyré, "Exact support recovery for sparse spikes deconvolution," Foundations of Computational Mathematics, vol. 15, no. 5, pp. 1315-1355, 2015.

[11] Q. Denoyelle, V. Duval, and G. Peyré, "Support recovery for sparse super-resolution of positive measures," Journal of Fourier Analysis and Applications, vol. 23, no. 5, pp. 1153-1194, 2017.

[12] M. Unser, J. Fageot, and J.P. Ward, "Splines are universal solutions of linear inverse problems with generalized TV regularization," SIAM Review, vol. 59, no. 4, pp. 769-793, 2017.

[13] A. Flinth and P. Weiss, "Exact solutions of infinite dimensional total-variation regularized problems," arXiv preprint arXiv:1708.02157, 2017.
[14] C. Dossal, V. Duval, and C. Poon, "Sampling the Fourier transform along radial lines," SIAM Journal on Numerical Analysis, vol. 55, no. 6, pp. 2540-2564, 2017.

[15] C. Aubel, D. Stotz, and H. Bölcskei, "A theory of superresolution from short-time Fourier transform measurements," Journal of Fourier Analysis and Applications, vol. 24, no. 1, pp. 45-107, 2018.

[16] H. Gupta, J. Fageot, and M. Unser, "Continuous-domain solutions of linear inverse problems with Tikhonov vs. generalized TV regularization," IEEE Transactions on Signal Processing, vol. 66, no. 17, pp. 4670-4684, 2018.

[17] I.J. Schoenberg, Cardinal Spline Interpolation, SIAM, Philadelphia, 1973.

[18] M. Unser, "Splines: A perfect fit for signal and image processing," IEEE Signal Processing Magazine, vol. 16, no. 6, pp. 22-38, November 1999.

[19] S. Boyd, N. Parikh, E. Chu, B. Peleato, and J. Eckstein, "Distributed optimization and statistical learning via the alternating direction method of multipliers," Foundations and Trends $\mathrm{R}$ in Machine Learning, vol. 3, no. 1, pp. 1-122, 2011.

[20] G. Dantzig, A. Orden, and P. Wolfe, "The generalized simplex method for minimizing a linear form under linear inequality restraints," Pacific Journal of Mathematics, vol. 5, no. 2, pp. 183-195, 1955.

[21] M. Unser, E. Soubies, F. Soulez, M. McCann, and L. Donati, "GlobalBioIm: A unifying computational framework for solving inverse problems," in Proceedings of the OSA Imaging and Applied Optics Congress on Computational Optical Sensing and Imaging (COSI'17), San Francisco CA, USA, June 26-29, 2017, paper no. CTu1B.

[22] E.J. Candès and T. Tao, "Near-optimal signal recovery from random projections: Universal encoding strategies?," IEEE Transactions on Information Theory, vol. 52, no. 12, pp. 5406-5425, 2006. 